\title{
New approach for consideration of adsorption/desorption data
}

\author{
Mihaela-Cristina Băleanu ${ }^{\mathrm{a}, \mathrm{b}}$, Raoul R. Nigmatullin ${ }^{\mathrm{c}, *}$, Salih Okur ${ }^{\mathrm{d}}$, Kasim Ocakoglu ${ }^{\mathrm{e}}$ \\ ${ }^{a}$ Faculty of Physics, University of Bucharest, P.O. Box MG-7, Măgurele 077125, Romania \\ ${ }^{\mathrm{b}}$ Mihail Sadoveanu Theoretical High School, District 2, Bucharest 021586, Romania

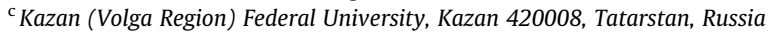 \\ d Izmir Institute of Technology, Faculty of Science, Izmir TR-35430, Turkey \\ e Advanced Technologies Research E Application Center, Mersin University, TR-33343 Yenisehir, Mersin, Turkey
}

\section{A R T I C L E I N F O}

\section{Article history:}

Available online 17 March 2011

\section{Keywords:}

QCM

Langmuir model

Eigen-Coordinates

Adsorption

Desorption

Ruthenium polypridyl complex

\begin{abstract}
A B S T R A C T
In this paper we proposed a new approach to modify the Langmuir model by considering nonlinear effects such as diffusion of water molecules in/out of an adsorbing film for humidity adsorption and desorption kinetics. The model was tested on the humidity adsorption and desorption data of a spin coated $50 \mathrm{~nm}$ thick Ruthenium polypridyl complex (Ru-PC K314) film, measured under relative humidity between $11 \%$ and $97 \%$ using by Quartz Crystal Microbalance (QCM) technique.
\end{abstract}

(C) 2011 Elsevier B.V. All rights reserved.

\section{Introduction}

Quartz Crystal Microbalance (QCM) has been frequently used investigate the kinetics of adsorption/desorption of adsorbate molecules [1,2] for monolayer films. It is a powerful technique to determine humidity sensing properties of materials before designing a sensor device during development stages. It is well known that the Langmuir model has been used successfully for monolayer films to analyze adsorption kinetics. A novel ceramic nanowires of TiO2 and poly (2-acrylamido-2methylpropane sulfonate) composite material films coated QCM was prepared as a low humidity sensor [3].

In the literature, the swelling behaviour of a series of hydrophilic random copolymer coatings in controlled humidity environments and in water has been investigated using QCM and spectroscopic ellipsometry by Chen et al. [4]. Thin films of polyaniline base, emeraldine base (EB), coating on the quartz crystal microbalance (QCM) electrode have been used as a sensitive layer for the detection of a number of primary aliphatic alcohols such as ethanol, methanol, 2-propanol and 1-propanol vapours [5]. A QCM coated with Nafion ${ }^{\circledR}$ film recast from Nafion ${ }^{\circledR}(\mathrm{Ag})$ complex solution has been used to investigate the interaction between methanol, water and $\mathrm{Nafion}^{\circledR}(\mathrm{Ag})$ and to determine simultaneously the concentration of methanol and water (relative humidity) [6].

In this work, we suggest a generalized Langmuir model to include nonlinear effect such as diffusion and condensation during humidity adsorption/desorption process. The suggested model was used to analyze the humidity adsorption and desorption QCM data of spin coated $50 \mathrm{~nm}$ Ruthenium polypridyl complex (Ru-PC K314) obtained under relative humidity between $11 \%$ and $97 \%$.

\footnotetext{
* Corresponding author.

E-mail addresses: cris_balance@yahoo.com (M.-C. Băleanu), nigmat@knet.ru (R.R. Nigmatullin), salihokuru@iyte.edu.tr (S. Okur), kasim.ocakoglu@ mersin.edu.tr (K. Ocakoglu).
} 


\section{QCM adsorption and desorption kinetics}

QCM has been used frequently to measure the fractional coverage $\theta$ as a function of time during the adsorption and desorption of water vapor molecules by Calix [4] arene films, while the increase in the frequency shift reflects the molecular mass uptake or loss $[7,8]$. Hence the difference between the oscillation frequency shift $(\Delta \mathrm{f})$ of coated and uncoated QCM is directly proportional to the adsorbed mass of moisture molecules. The simplest relationship between the surface adsorption kinetics and frequency shift $(\Delta \mathrm{f})$ of QCM can be expressed as following:

$$
\frac{d \Delta f}{d t}=-\left(K_{a} C+K_{d}\right) \Delta f+K_{a} C \Delta f_{\max }
$$

where $\Delta f$ is the QCM resonance frequency, $t$ is time, $K_{a}$ and $K_{d}$ are the adsorption and desorbtion rate constants and $C$ is the water vapor concentration in the air. During the adsorption process, $\Delta f$ is equal to $\Delta f_{\max }$ for very long time.

\section{Experimental}

Gold coated QCM quartz crystal electrodes were placed into ethanol and ultrasonically cleaned, then rinsed by de-ionized water. $1 \mathrm{mg} / \mathrm{ml} \mathrm{Ru-PC}$ was dissolved in deionized water. $5 \mu \mathrm{l}$ of solution was spin-coated onto quartz crystal with $2000 \mathrm{rpm}$. After drying at room conditions, it was kept in dessicator at room temperature for $3 \mathrm{~h}$. Then the quartz crystal coated with Ru-PC film was used to record both the reference frequency at $11 \%$ and the frequency changes up to $97 \%$ relative humidity. The thicknesses of films were measured using a Dektak profilometer from Veeco and found to be $50 \mathrm{~nm}$ [9].

A closed box, partly filled with saturated salt solutions, generated a humid environment with good accuracy in the free room above the salt was used to provide a fixed point humidity as shown in Fig. 1. The value of the relative humidity depends on the type of salt given in Ref. [7]. The same experimental setup given in our previous work [7] was used to measure the adsorption kinetics of Ru-PC films above saturated $\mathrm{LiCl}(11 \% \mathrm{RH})$ and $\mathrm{K} 2 \mathrm{SO} 4(97 \% \mathrm{RH})$ aquatic solution level inside a half filled closed container. (Fig. 2) shows the chemical structure of the substance used in mesurements.

A Time-Resolved Electrochemical Quartz Crystal Microbalance (EQCM) with the model of CHI400A Series from CH Instruments (Austin, USA) has been used to measure the change in the resonance frequency of quartz crystals between gold electrodes via both serial and usb interface connected to a computer. The QCM works with oscillation frequencies between 7.995 MHz-7.950 MHz. The density $(\rho)$ of the crystal is $2.684 \mathrm{~g} / \mathrm{cm}^{3}$, and the shear modulus $(\mu)$ of quartz is $2.947 \times 10^{11} \mathrm{~g} / \mathrm{cm} \cdot \mathrm{s}^{2}$. Around oscillation frequency of $7.995 \mathrm{MHz}$, a net change of $1 \mathrm{~Hz}$ corresponds to $1.34 \mathrm{ng}$ of materials adsorbed or desorbed onto the crystal surface of an area of $0.196 \mathrm{~cm}^{2}$.

The signals coming from a QCM electrode and a commercial RH humidity sensor were simultaneously measured during the adsorption and desorption process. Both the relative humidity and temperature were also recorded during measurements while maintaining the temperature around $23^{\circ} \mathrm{C}$. For this purpose, a EI-1050 selectable digital relative humidity

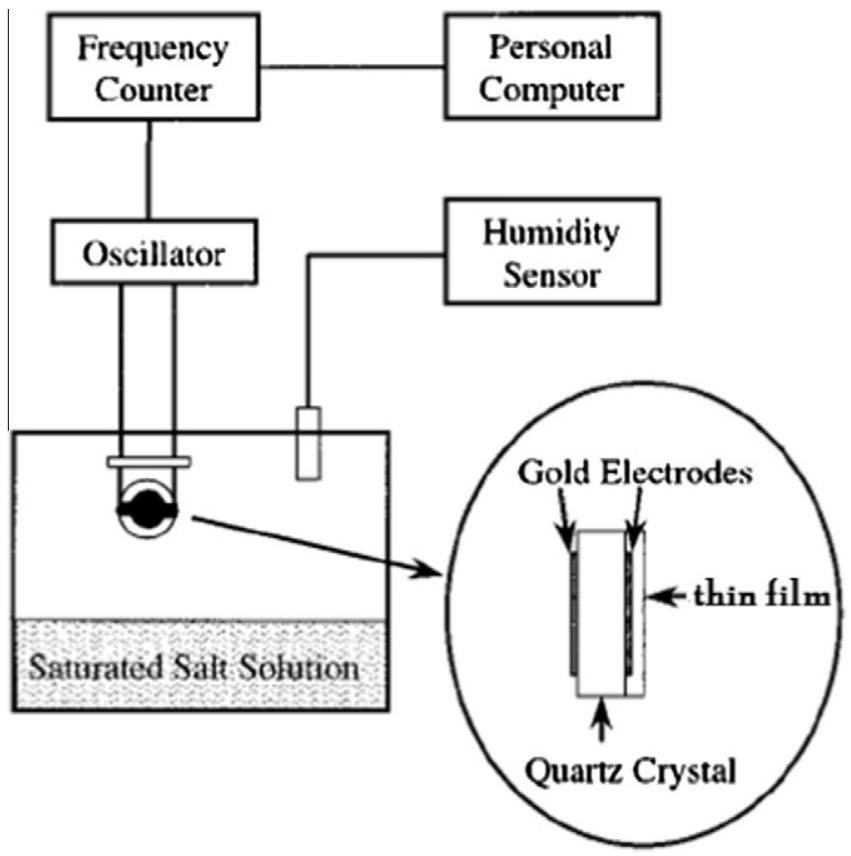

Fig. 1. The experimental setup to measure the adsorption and desorption kinetics of Ru- 314 films under fixed point humidity between $25 \%$ RH and $80 \%$ RH above a saturated salt solution inside a closed container using hybrid system of QCM electrodes and a commercial humidity sensor. 


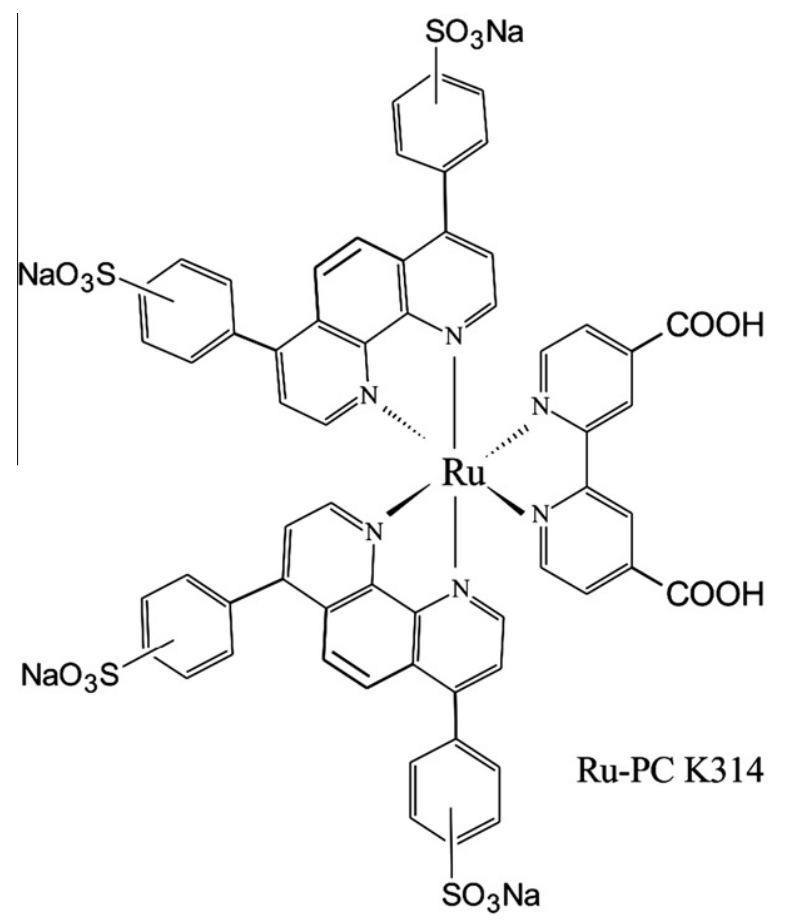

Fig. 2. Chemical structure of ruthenium polypridyl complex.

and temperature probe with a response time of $4 \mathrm{~s}$ and a resolution of $0.03 \% \mathrm{RH}$ was used with a USB controlled LabJack U12 ADC system combined with a single chip sensor module (SHT11) manufactured by Sensirion (Staefa, Switzerland). The detailed information about the synthesis of [RuII (bis (4; 7-diphenyl-1; 10-phenanthroline-disulfonic acid disodium salt) (4; 4'dicarboxy-2;2'-bipyridine)], (Ru-PC K314) can be found in Ocakoglu and Okur et al. [10].

\section{Langmuir model and its modification}

In this paper, we deal with Langmuir model which was first developed in 1916 by Irving Langmuir, [11] and developed later by others $[12,13]$. In order to analyze the adsorption and desorption kinetics of gas vapor molecules onto organic or inorganic films, Langmuir adsorption isotherm model is applied by [14-18]. As a result the model describes the rate of surface reaction for forming a monolayer on the surface by using the below equation,

$$
\frac{d \theta}{d t}=k_{a}(1-\theta) C-k_{d} \theta
$$

Here $\theta$ is a unitless quantity, e.g. fraction of surface coverage, $K_{a}$ and $K_{d}$ denote the rate constants for the adsorption and desorption processes. In this experiment, the concentration $C$ is proportional to the relative humidity.

In this study QCM has been used to measure the fractional coverage $\theta$ a function of time during the adsorption and desorption of water vapor molecules.

Hence the difference between the oscillation frequency shifts $\Delta f$ of coated and uncoated QCM is directly proportional to the adsorbed mass of moisture molecules. The relationship between the surface adsorption kinetics and frequency shift $(\Delta f)$ of QCM can be expressed by expression (2.1).

As one can notice from comparison of expressions (2.1) and (4.1), the frequency shift $\Delta f$ is directly proportional to $\theta$. The fitting of experimental data realized with accordance of expression (4.1) by the Eigen-Coordinates (ECs) method gives unsatisfactory results [19]. We see at least two possible reasons: (a) experimental data are not so clean and always contains some uncontrollable factors; (b) the experimental data contains more than one exponential function and so the simplest model (4.1) should be modified and generalized. In order to see possible modifications let us present Eq. (4.1) in the form of the finite difference equation:

$$
\theta(t+T) \cong T K_{a}+\left[1-T\left(K_{a}+K_{d}\right)\right] \theta
$$

Eq. (2.1) can be rewritten in complete analogy with Eq. (4.2).

Eq. (4.2) expresses in the simplest form the linear principle of the strongly-correlated variables. Eq. (4.2) can be easily generalized if we present this equation in the form: 


$$
F(t+k T)=\sum_{n=0}^{k-1} a_{n} F(t+n T)+c_{0}
$$

This equation has a simple physical meaning. It signifies that the adsorption/desorption phenomenon can be divided on some stages related on a structure of a surface (which in the most cases represents a random fractal surface) and the geometrical configuration of the adsorbed/desorbed molecules. The physical meaning of the constants $a_{n}$ should be related with relative concentrations of the molecules and character of the surface that participate in this phenomenon. Their exact dependence on concentration and fractal dimension of the surface considered will be a subject of the further research. In this paper we only verify this general relationship on available data. The solution of the functional equation was considered in paper [20]. It is expressed in the form of linear combination of exponential functions:

$$
F(t)=\sum_{n=1}^{k} E_{n}(t) \exp \left(\lambda_{n} t\right)+b
$$

Here the constant $b$ is expressed in the form

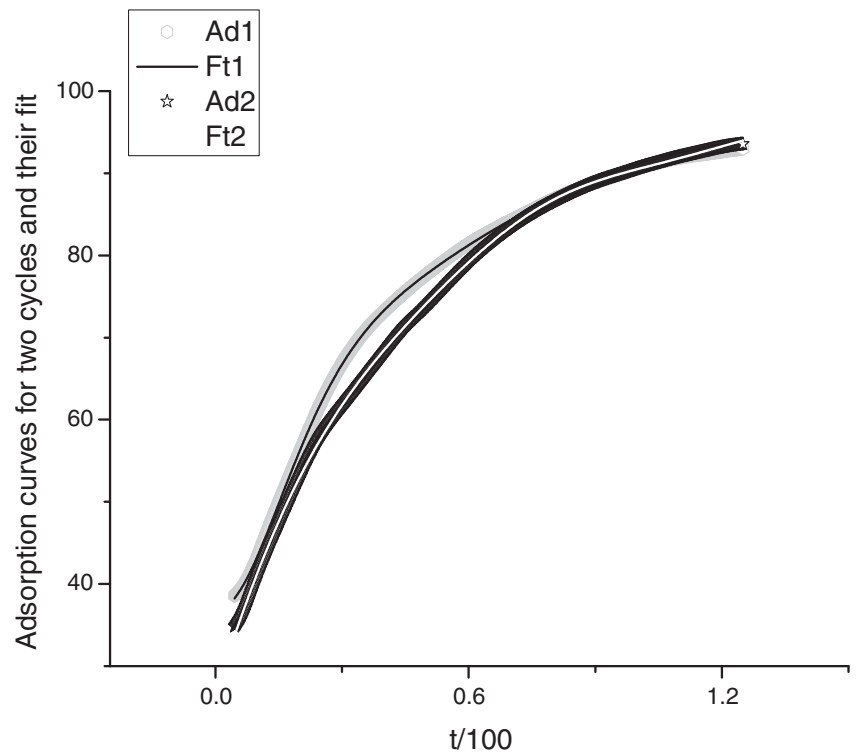

Fig. 3. The fit of the adsorption curves realized for two cycles with function (4.8).

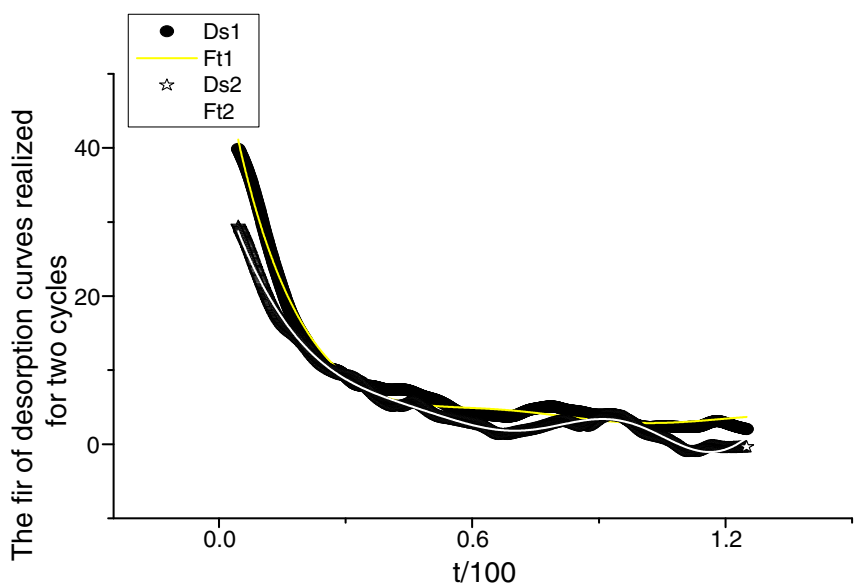

Fig. 4. The fit of desorption curves realized for two cycles with function (4.8). 
Table 1

The fitting parameters presented by (4.8)

\begin{tabular}{lllc}
\hline Cycle & $A_{0}, A_{1}$ & $A_{2}, A_{3}$ & $\lambda_{1}, \lambda_{2}$ \\
\hline Ad1 & $96.971,-0.9397$ & $-13.247,-59.227$ & $-6.8904,-2.2149$ \\
Ad2 & $98.647,0.2634$ & $0.69817,-71.822$ & $-0.54768,-2.1982$ \\
Ad3 & $97.677,3.1639$ & $8.325,-93.886$ & $-5.5395,-2.6999$ \\
Des1 & $3.4147,0.86231$ & $-0.33054,50.218$ & $-0.41477,-6.7359$ \\
Des2 & $1.664,0.05576$ & $-0.12518,34.386$ & 10.206 \\
Des3 & $2.0239,3.2448$ & 2.363158 .115 & 8.6866 \\
\hline
\end{tabular}

Ad $=$ Adsorption, Des $=$ Desorption .

$$
b=c_{0}\left(1-\sum_{n=0}^{k-1} a_{n}\right)^{-1}
$$

The unknown exponential constants $\lambda_{n}$ are found as roots of the characteristic polynomial

$$
x^{k}-\sum_{n=0}^{k-1} a_{n} x^{n}=0, \quad \lambda_{m}=\frac{\ln \left(x_{m}\right)}{T}, \quad m=1,2, \ldots, k
$$

$E_{n}(t)$ in (4.4) can be expressed approximately in the form of periodical functions with period $T$.

$$
E_{n}(t) \cong a_{n}+b_{n} \cos \left(2 \pi\langle m\rangle \frac{t}{T}-\varphi_{n}\right)
$$

Here $a_{n}, b_{n}$ and $\varphi_{n}$ are some fitting constants. The value $\langle m\rangle$ defines the leading mode. The fitting to function (4.4) can be realized with the help of the ECs method [19] that helps to reduce the non-linear fitting procedure to the conventional linear least square method (LLSM). For verification of the hypothesis (4.3) we chose the minimal number of components (items) $k=3$. The fitting function identified by the ECs method is written as:

$$
\theta(t)=A_{0}+A_{1} \cos (\omega t) e^{\lambda_{1} t}+A_{2} \sin (\omega t) e^{\lambda_{1} t}+A_{3} e^{\lambda_{2} t}
$$

This fitting function (4.8) contains 6 fitting parameters and describes all available data with acceptable accuracy. Figs. 3 and 4. Show the fit to the adsorption and desorption data for two different cycles, respectively.

The set of the fitting parameters are given in Table 1. Notations are taken from expression (4.8). The accuracy of adsorption data is located in the interval: $[0.04 \%<\operatorname{RelErr}(\%)<0.07 \%]$ as for the desorption data the accuracy is in the interval. $[0.15 \%<\operatorname{RelErr}(\%)<0.34 \%]$.

\section{Conclusions}

Finding new generalization of the Langmuir model is an interesting problem. In this work, a generalized Langmuir model including nonlinear effect such as diffusion and condensation during humidity adsorption/desorption process was used to analyze the humidity adsorption and desorption QCM data of spin coated $50 \mathrm{~nm}$ Ruthenium polypridyl complex (Ru-PC K314) obtained under relative humidity between $11 \%$ and $97 \%$. The obtained results definitely show that the Langmuir equations can be generalized by making use of the ECs method. The experimental confirmation of the hypothesis (4.8) that follows from solution of the functional Eq. (4.3) implies that some important factors participating in adsorption/desorption phenomena can be taken into account. This is the first and serious generalization attempt of such kind that received the experimental confirmation. The analysis of the physical meaning of the parameters $a_{n}$ that figuring in Eq. (4.3) and the proper explanation of oscillating solutions merits a special research.

\section{References}

[1] Hartmann J, Auge J, Lucklum R, Rosler S, Hauptmann P, Adler B. Supramolecular interactions on mass sensitive sensors in 261 gas phases and liquids. Sens Actuat B: Chem 1996;34:305-11.

[2] Kalchenko VI, Koshets IA, Matsas EP, Kopylov ON, Solovyov A, Kazant ZI, et al. Calixarene-based qcm sensors array and its response to 265 volatile organic vapours. Mater Sci 2002;20(3):73-88.

[3] Su P-G, Sun Y-L, Lin C-C. Novel low humidity sensor made of TiO2 nanowires/poly(2-acrylamido-2-methylpropane sulfonate) composite material film combined with quartz crystal microbalance. Talanta 2006;69:946951.

[4] Chen W-L, Shull KR, Papatheodorou T, Styrkas DA, Keddie JL. Equilibrium swelling of hydrophilic polyacrylates in humid Environments. Macromolecules 1999;32:136-44.

[5] Ayad MM, El-Hefnawey G, L Torad N. A sensor of alcohol vapours based on thin polyaniline base film and quartz crystal microbalance. J Hazard Mat 2009; 168:8588-97.

[6] Sun L-X, Okada T. Simultaneous determination of the concentration of methanol and relative humidity based on a single Nafion (Ag)-coated quartz crystal microbalance. Anal Chim Acta 2000;421:8392-9.

[7] Okur S, Kus Mç, Özel F, Aybek V, Yilmaz M. Humidity adsorption kinetics of Calix [4] arene derivatives measured using QCM. Talanta 2010;81/1$2: 248-51$. 
[8] Okur S, Kus M, Özel F, Yilmaz M. Humidity adsorption kinetics of water soluble Calix [4] arene derivatives measured using QCM technique. Sens Actuat B: Chem 2010;145/1:93-7.

[9] Baleanu D, Okur Yeliz Y, Okur S, Ocakoglu K. Nonlinear dynamics of complex systems: applications in physical. In: Tenreiro Machado JA, Baleanu D, Luo A, editors. Biological and financial systems. Springer; 2011.

[10] Ocakoglu K, Okur S. Humidity sensing properties of novel ruthenium polypridyl complex. Sens Actuat B: Chem 2010;151/1:223-8.

[11] Langmuir I. The constitution and fundamental properties of solids and liquids. J Am Chem Soc 1916;38:2221-95.

[12] Kankare J, Vinokurov IA. Kinetics of Langmuirian adsorption onto planar, spherical, and cylindrical surfaces. Langmuir 1999;15:5591-9.

[13] Kapoor A, Ritter JA, Yang RT. An extended Langmuir model for adsorption of gas mixtures on heterogeneous surfaces. Langmuir 1990;6(3):660-4.

[14] Sauerbrey G. Verwendung von schwingquarzen zur wagung dunner schichten und zur mikrowagung. Z Phys 1959;155:206-22.

[15] Karpovich DS, Blanchard GJ. Direct measurement of the adsorption kinetics of alkanethiolate self-assembled monolayers on a microcrystalline gold surface. Langmuir 1994;10:3315-22.

[16] Gregg SJ, Sing KSW. Adsorption, surface area and porosity. Academic Press; 1967.

[17] Sun YL, Wu RJ, Huang YC, Su PG, Chavali M, Chen YZ, et al. In situ prepared polypyrrole for low humidity qcm sensor and related theoretical calculation. Talanta 2007;73(151):857-61.

[18] Su PG, Chang YP. Low humidity sensor based on a quartz-crystal microbalance coated with polyprrole/Ag/TiO 2 nanoparticles composite thin films. Sens Actuat B: Chem 2008;125:915-20.

[19] Nigmatullin RR. Eigen-coordinates: new method of identification of analytical functions in experimental measurements. Appl Magn Reson 1998;14:601-33.

[20] Nigmatullin RR. Strongly correlated variables and existence of the universal distribution function for relative fluctuations. Phys Wave Phen 2008;16:119-45. 